\title{
Hemostatic Efficacy and Safety of the Hemostatic Powder UI-EWD in Patients with Lower Gastrointestinal Bleeding
}

\section{Boram Cha}

Inha University School of Medicine

\section{Dong Hyun Lee}

Inha University School of Medicine

Jongbeom Shin ( $\nabla$ shinjongv@naver.com )

Inha University School of Medicine

Jin-Seok Park

Inha University School of Medicine

\section{Kye Sook Kwon}

Inha University School of Medicine

\section{Hyungkil Kim}

Inha University School of Medicine

\section{Research Article}

Keywords: Lower gastrointestinal bleeding, hemostatic UI-EWD powder, re-bleeding, safety

Posted Date: December 20th, 2021

DOI: https://doi.org/10.21203/rs.3.rs-1117741/v1

License: (1) This work is licensed under a Creative Commons Attribution 4.0 International License.

Read Full License

Version of Record: A version of this preprint was published at BMC Gastroenterology on April 7th, 2022. See the published version at https://doi.org/10.1186/s12876-022-02247-4. 


\section{Abstract}

Background and Aims: Acute lower gastrointestinal bleeding (LGIB) is a common cause of emergency hospitalization and may require readmission for re-bleeding. Recently, a novel endoscopic hemostatic powder (UI-EWD/Nexpowder ${ }^{\mathrm{TM}}$, Nextbiomedical, Incheon, South Korea) was developed and applied for the control of LGIB. The aim of this study was to evaluate the hemostatic efficacy and long-term safety of UIEWD in LGIB.

Patients and Methods: We conducted a retrospective cohort study of LGIB at a single tertiary center in south korea. One hundred and sixty-seven consecutive patients with LGIB who were initially successful in endoscopic hemostasis were included and divided into the conventional treatment group $(n=112)$ and the UI-EWD therapy group ( $n=55 ; 38$ patients with conventional treatment and 17 patients with UI-EWD alone). The success rate of hemostasis, adverse events related to UI-EWD, and re-bleeding rate were evaluated.

Results: The incidence of endoscopic hemostasis applied to the hepatic flexure (7.3\% vs. $0 \%, p=0.011)$ and larger than $4 \mathrm{~cm}(25.5 \%$ vs. $8.0 \%, p=0.002)$ were significantly higher in the UI-EWD group than in the conventional therapy group. The cumulative rebleeding rate within 28 days in the UI-EWD group was 5.5\% (3/55), which was significantly lower than that in the conventional treatment group $(17.0 \%$ [19/112]; $p=$ 0.039). No UI-EWD-related adverse events were recorded.

Conclusion: Based on our results, application of UI-EWD in LGIB showed promising results for the prevention of re-bleeding, especially in locations where it is difficult to approach or cases with more bleeding. There were no significant complications, such as perforation or embolism. In particular, UI-EWD should be considered first for anatomical or technical impediments to endoscopic access in LGIB.

\section{Introduction}

Low gastrointestinal bleeding (LGIB) accounts for $20-25 \%$ of all gastrointestinal (GI) bleeding episodes $[1,2]$. Although LGIB is relatively less common than upper GI bleeding, it remains a frequent cause of hospitalization, with a mortality rate of up to $20 \%[3,4]$. Despite high success in the initial hemostasis of LGIB, the re-bleeding rate is still high, up to $16.8 \%$ [5]. Moreover, limitations remain despite the recent development of endoscopic hemostasis technology, including cautery, thermocoagulation, clip, injection therapy, and argon plasma coagulation [6]. Considering the high risk of perforation due to the thinner wall of the colon compared to the stomach, endoscopists tend to be limited in their endoscopic hemostasis, especially in LGIB. Indeed, hemoclips are not suitable for diffuse bleeding lesions and can cause mechanical mucosal damage by the device. Although thermocoagulation is suitable for large bleeding surfaces, endoscopists need to consider coagulation syndrome and delayed perforation $[1,7]$.

To compensate for these limitations, hemostatic powders such as Hemospray (also known as TC-325) (Cook Medical, Winston-Salem, NC, USA) [8, 9], EndoClot (AMP; EndoClot Plus Inc., Santa Clara, CA, USA) $[10,11]$, and ABS (Ankaferd Health Products Ltd., Istanbul, Turkey) [12] have been developed as 
alternative non-contact and non-traumatic endoscopic tools, especially for diffuse and large bleeding lesions, with excellent initial hemostatic rates of up to $98 \%$ in upper $\mathrm{GI}$ bleeding $[13,14]$. Moreover, previous reports have also shown the high hemostatic efficacy of hemostatic powders in LGIB [15-17].

Although the effects of lowering the rebleeding rate of hemostatic powders on upper and lower gastrointestinal bleeding have been reported, even with the addition of hemostatic powders, higher rebleeding rates of up to $13 \%$ in LGIB powders remains a challenge for endoscopists [16]. A highly adhesive hemostatic powder (UI-EWD, Nextbiomedical, Incheon, South Korea), which is a biocompatible natural polymer consisting of aldehyde dextran and succinic acid modified $\varepsilon$-poly, has been newly developed. Recently, good hemostatic efficacy of UI-EWD on refractory upper GI bleeding, non-variceal bleeding, and diffuse tumor bleeding, with low rebleeding rates was reported [18-22]. However, no previous study has examined the hemostatic effects of UI-EWD in patients with LGIB.

We aimed to retrospectively evaluate the hemostatic efficacy and safety of UI-EWD in patients with LGIB.

\section{Patients And Methods}

\section{Study design and study population}

Patients treated for LGIB between January 2017 and July 2021 at Inha University Hospital were enrolled. The patients were retrospectively selected from established prospective registries based on the following inclusion criteria: (1) age > 18 years at the time of treatment; (2) suspected active LGIB (e.g., hematochezia or melena); and (3) past endoscopic hemostasis. The exclusion criteria were as follows: (1) upper GI bleeding; (2) hematologic disorder; (3) autoimmune disease; (4) pregnancy or suspected pregnancy at the time of treatment; and (5) receipt of treatment by other endoscopic or surgical treatment within 30 days prior to UI-EWD application. Finally, 167 patients met the inclusion and exclusion criteria (Fig.1). The medical records of the included patients were reviewed. Information on clinical characteristics, bleeding, clinical outcomes (including immediate hemostasis success and re-bleeding rates), and hemostasis-related complications, including UI-EWD-associated adverse events, were collected.

\section{Endoscopic procedures}

UI-EWD was applied to LGIB using a conventional endoscope (H290-TL/I, Olympus, Tokyo, Japan) by experienced endoscopists (6-25 years). In the UI-EWD group, the final hemostasis sequence was UI-EWD. UI-EWD was used as monotherapy or rescue therapy when the endoscopist determined the most appropriate time to apply UI-EWD to LGIB. UI-EWD monotherapy was applied when bleeding was minimal and was enough for the single use of UI-EWD or on diffuse oozing bleeding that might not be treated effectively by conventional therapy. Rescue therapy was defined as additional treatment when continuous bleeding was observed, even after conventional treatment. UI-EWD was sprayed onto the surface of the bleeding site using a catheter passed through the powder delivery system (Fig.2) under 
direct endoscopic vision until the bleeding lesion was completely covered with the powder (Fig.3). UI-EWD was applied with a maximum release of $6 \mathrm{~g}$ of powder.

\section{Outcome measurements}

Successful initial hemostasis was defined as confirmation of hemostasis until 5 min after the endoscopic treatment. If bleeding persisted after 5 min with conventional hemostasis, UI-EWD was applied as a rescue therapy at the endoscopist's discretion. Immediate hemostasis failure was defined as cases in which additional application of treatment modalities was required after applying UI-EWD. Rebleeding was defined as clinical evidence of bleeding, such as melena or hematemesis, with an associated reduction of $2 \mathrm{~g} / \mathrm{dL}$ of hemoglobin within 28 days after the initial successful endoscopic hemostasis [23]. When re-bleeding was suspected, further endoscopic evaluation was performed to confirm the actual bleeding status. The patients' medical records were reviewed to assess the adverse events associated with UI-EWD, such as newly developed symptoms (e.g., abdominal pain, nausea, and vomiting), changes in vital signs, detection of free air on abdominal plain radiography, and laboratory test abnormalities. Moreover, the possibility of embolism, intestinal obstruction, and allergic reactions due to the characteristics of the powder were determined.

\section{Statistical analysis}

The clinical characteristics of the study subjects were expressed as medians (ranges) for continuous variables and numbers (percentages) for categorical variables. The differences between categorical or continuous variables were analyzed using the Mann-Whitney U test, Student's t-test, chi-square test, or Fisher's exact test. The overall re-bleeding and cumulative survival rates were estimated using the Kaplan-Meier method. Two-tailed p-values $<0.05$ were considered statistically significant. Statistical analyses were performed using SPSS v25.0 (SPSS Inc., Chicago, IL, USA).

\section{Results}

\section{Baseline clinical characteristics of the conventional group and the UI-EWD therapy group}

Between March 2017 and July 2021, 192 patients were treated for LGIB. In one patient, treatment was not possible because of excessive bleeding, and 25 patients were excluded based on the exclusion criteria. Finally, 167 patients were enrolled in the study. The baseline clinical characteristics of the conventional treatment and UI-EWD therapy patients are shown in Table 1. Conventional therapy for lower GI bleeding was performed in 112 patients, and UI-EWD was applied during endoscopic treatment in 56 patients. UIEWD was used as a monotherapy in 17 patients (30.9\%) and as a rescue treatment in 38 patients $(69.1 \%)$ (Fig. 1). There was no significant difference in age $(74(39-88)$ vs. $64(25-89), p=0.18)$, percentage of 
male patients $(58.9 \%$ vs. $58.2 \%, p=0.88)$, or percentage of treatment with anticoagulants $(8.0 \%$ vs. $5.5 \%$, $p=0.75)$ or antithrombotic agents $(28.6 \%$ vs. $16.4 \%, p=0.09)$ or both $(9.8 \%$ vs. $9.1 \%, p=0.55)$ between the conventional and UI-EWD groups. Moreover, the two groups were similar in terms of laboratory findings (Table 1). The median follow-up duration was not significantly different between the conventional and UI-EWD groups (131 (7-668) vs. 47 (7-797), p = 0.67).

Table 1

Baseline clinical characteristics of study subjects.

\begin{tabular}{|c|c|c|c|}
\hline Variables & $\begin{array}{l}\text { Conventional therapy } \\
(n=112)\end{array}$ & $\begin{array}{l}\text { UI-EWD therapy } \\
(n=55)\end{array}$ & P-value \\
\hline Age (year)§ & $74(39-88)$ & $64(25-89)$ & 0.181 \\
\hline Sex (male) $)^{\S}$ & $66(58.9)$ & $32(58.2)$ & 0.875 \\
\hline \multicolumn{4}{|l|}{ Medication, n (\%) } \\
\hline Anticoagulant & $9(8.0)$ & $3(5.5)$ & $0.753^{+}$ \\
\hline Antiplatelet agent & $32(28.6)$ & $9(16.4)$ & 0.085 \\
\hline Anticoagulant and antiplatelet agent & $11(9.8)$ & $5(9.1)$ & $0.549^{+}$ \\
\hline White blood cell $(1000 / \mu \mathrm{L})$ & $6580(2100-20570)$ & $7270(2290-36410)$ & 0.337 \\
\hline $\mathrm{Hb}(\mathrm{g} / \mathrm{dL})^{\S}$ & $10.0(12.0-4.9)$ & $12.4(6.4-16.0)$ & 0.369 \\
\hline Platelet count $(1000 / \mu \mathrm{L})$ & $208(55-654)$ & $222(49-371)$ & 0.550 \\
\hline PTs (INR) & $1.03(0.84-5.18)$ & $1.05(0.84-18.40)$ & 0.320 \\
\hline aPTT (s) & $36.5(24.2-79.2)$ & $36.3(26.2-79.2)$ & 0.457 \\
\hline BUN (mg/dL) & $16.4(5.5-139.8)$ & $15.5(6.5-95.3)$ & 0.372 \\
\hline $\mathrm{Cr}(\mathrm{mg} / \mathrm{dL})$ & $0.87(0.29-8.43)$ & $0.83(0.29-7.28)$ & 0.502 \\
\hline Follow-up duration (day) $\S$ & $131(7-668)$ & $47(7-797)$ & 0.674 \\
\hline \multicolumn{4}{|c|}{$\begin{array}{l}\text { Abbreviations: UGI: Upper gastrointestinal, LGI: Lower gastrointestinal, Hb: Hemoglobin, PTs: } \\
\text { Prothrombin time, aPTT: Activated partial thromboplastin time, BUN: Blood urea nitrogen, Cr: } \\
\text { Creatinine. }\end{array}$} \\
\hline \multicolumn{4}{|l|}{ §Median (range) } \\
\hline \multicolumn{4}{|l|}{${ }^{+}$Fisher's exact test } \\
\hline
\end{tabular}




\section{Bleeding characteristics of the conventional group and the UI-EWD therapy group}

The bleeding characteristics of the study subjects are shown in Table 2. The UI-EWD group was significantly more applied to the hepatic flexure $(7.3 \%$ vs. $0 \%, p=0.01)$ and slightly more applied to the sigmoid colon $(18.2 \%$ vs. $11.6 \%, p=0.25)$. 
Table 2

Bleeding characteristics of the study subjects

\begin{tabular}{|c|c|c|c|}
\hline Variables & $\begin{array}{l}\text { Conventional therapy } \\
(n=112)\end{array}$ & $\begin{array}{l}\text { UI-EWD therapy } \\
(n=55)\end{array}$ & P-value \\
\hline \multicolumn{4}{|l|}{ Location of bleeding, $\mathrm{n}(\%)$} \\
\hline Cecum & $5(4.5)$ & $2(3.6)$ & 0.625 \\
\hline Ascending colon & $18(16.1)$ & $5(9.1)$ & 0.219 \\
\hline Hepatic flexure & $0(0.0)$ & $4(7.3)$ & $0.011^{+}$ \\
\hline Transverse colon & $12(10.7)$ & $4(7.3)$ & 0.478 \\
\hline Descending colon & $5(4.5)$ & $1(1.8)$ & 0.388 \\
\hline Sigmoid colon & $13(11.6)$ & $10(18.2)$ & 0.247 \\
\hline Rectum & $59(52.7)$ & $29(52.7)$ & 0.830 \\
\hline \multicolumn{4}{|l|}{ Cause of bleeding, $\mathrm{n}(\%)$} \\
\hline Diverticular bleeding & $13(11.6)$ & $4(7.3)$ & 0.204 \\
\hline Radiation proctitis & $13(11.6)$ & $1(1.8)$ & $0.037^{+}$ \\
\hline Angiodysplasia & $9(8.0)$ & $1(1.8)$ & $0.168^{+}$ \\
\hline Ulcer bleeding & $34(30.4)$ & 18 (32.7) & 0.756 \\
\hline Post procedure bleeding & $31(27.7)$ & $25(45.5)$ & $0.147^{+}$ \\
\hline Tumor bleeding & $12(10.7)$ & $6(10.9)$ & 0.970 \\
\hline \multicolumn{4}{|l|}{ Size of bleeding site, $\mathrm{n}(\%)$} \\
\hline$<1 \mathrm{~cm}$ & $71(63.4)$ & $15(27.3)$ & $<0.001$ \\
\hline $1-4 \mathrm{~cm}$ & $32(28.6)$ & $26(47.3)$ & 0.022 \\
\hline$>4 \mathrm{~cm}$ & $9(8.0)$ & $14(25.5)$ & 0.002 \\
\hline \multicolumn{4}{|c|}{ Forrest classification, n (\%) } \\
\hline la & $2(1.8)$ & $5(9.1)$ & $0.040^{+}$ \\
\hline $\mathrm{lb}$ & $112(94.6)$ & $50(90.1)$ & $0.001^{+}$ \\
\hline
\end{tabular}

${ }^{+}$Fisher exact test

${ }^{*} P$-values were calculated using the $t$-test or Fisher calculated using the of the conventional therapy group and the UI-EWD therapy group 


\begin{tabular}{|llll|}
\hline Variables & $\begin{array}{l}\text { Conventional therapy } \\
(\mathbf{n}=\mathbf{1 1 2})\end{array}$ & $\begin{array}{l}\text { Ul-EWD therapy } \\
(\mathbf{n = 5 5 )}\end{array}$ & P-value \\
\hline Treatment modality, $\mathrm{n}(\%)$ & & $22(40.0)$ & 0.397 \\
\hline Coagrasper & $38(33.0)$ & $5(9.1)$ & 0.106 \\
\hline APC & $21(18.8)$ & $21(38.2)$ & $<0.001$ \\
\hline Hemoclipping & $81(53.6)$ & $0(0.0)$ & 0.156 \\
\hline EVL & $4(3.6)$ & $17(30.9)$ & \\
\hline UI-EWD only & $0(0.0)$ & & \\
\hline${ }^{+}$Fisher exact test & & \\
\hline${ }^{*} P$-values were calculated using the $t$-test or Fisher calculated using the of the conventional therapy \\
group and the Ul-EWD therapy group
\end{tabular}

Differences in the cause of bleeding between the two groups were evaluated. Radiation proctitis was significantly more common in the conventional therapy group $(11.6 \%$ vs. $1.8 \%, p=0.037)$ due to the higher use of argon plasma coagulation.

Bleeding sites $>1 \mathrm{~cm}$ (including more than $4 \mathrm{~cm}$ ) were significantly more common in the UI-EWD therapy group than in the conventional therapy group $(1-4 \mathrm{~cm}: 47.3 \%$ vs. $28.6 \%, p=0.02,>4 \mathrm{~cm}: 25.5 \%$ vs. $8.0 \%$, $p<0.001)$.

According to the initial status of bleeding, for Forrest lb, diffuse oozing bleeding, hemostasis with conventional therapy seemed sufficient $(94.6 \%$ vs. $90.1 \%, p=0.001)$; otherwise, additional UI-EWD treatment was significantly more applied to Forrest la, active spurting bleeding $(9.1 \%$ vs. $1.8 \%, p=0.04)$.

There was no significant difference in treatment modalities between the two groups, with the exception of hemoclipping, which was significantly more common in the conventional therapy group $(53.6 \%$ vs. $39.3 \%$, $\mathrm{p}<0.001)$.

\section{Comparison of clinical outcomes after bleeding control between the conventional group and the UI-EWD therapy group}

All patients in both groups showed successful immediate hemostasis. However, the cumulative rebleeding rate within 28 days was significantly lower in the UI-EWD therapy group $(5.5 \%$ vs. $17 \%, p=$ 0.04) (Table 3 and Fig. 4). The main difference in rebleeding rate between the two groups was determined within 7 days $(12.5 \%$ vs. $5.5 \%, p=0.12)$, and the re-bleeding rate was lower after 7 days in both groups. Moreover, the UI-EWD group did not show re-bleeding after 7 days. The median time to rebleeding was 
within 7 days, 5 days (1-25) in the conventional therapy group, and 4 days $(1-5)$ in the UI-EWD therapy group with no significant difference.

Table 3

Clinical outcomes of bleeding control

\begin{tabular}{|llll|}
\hline Variables & $\begin{array}{l}\text { Conventional therapy } \\
(\mathbf{n}=112)\end{array}$ & $\begin{array}{l}\text { Ul-EWD therapy } \\
(\mathbf{n}=\mathbf{5 5})\end{array}$ & P-value \\
\hline Success of immediate hemostasis, $\mathrm{n}(\%)$ & $112(100 \%)$ & $55(100 \%)$ & $<0.001$ \\
\hline Cumulative re-bleeding, $\mathrm{n}(\%)$ & & $3(5.5)$ & 0.157 \\
\hline At 7 days & $14(12.5)$ & $3(5.5)$ & 0.091 \\
\hline At 14 days & $16(14.3)$ & $3(5.5)$ & 0.039 \\
\hline At 28 days & $19(17.0)$ & $4(1-5)$ & - \\
\hline Time to re-bleeding (days) ${ }^{\S}$ & $5(1-25)$ & $0(0.0)$ & - \\
\hline Adverse event, $n$ (\%) & & $0(0.0)$ & - \\
\hline Colonic obstruction & $0(0.0)$ & $0(0.0)$ & \\
\hline Perforation & $1(0.9)$ & $0(0.0)$ & \\
\hline Infection & $1(0.9)$ & & \\
\hline Embolization & $0(0.0)$ & & \\
\hline SMedian (range) & & & \\
\hline+
\end{tabular}

There were no reported adverse events associated with UI-EWD application; otherwise, one patient in the conventional therapy group showed perforation with infection after hemostasis by hemoclipping (Table 3).

\section{Bleeding characteristics and clinical outcomes of the UI- EWD monotherapy group}

Of the 55 patients in the UI-EWD therapy group, 17 (30.9\%) received UI-EWD as monotherapy. We performed a subgroup analysis of bleeding characteristics and clinical outcomes according to UI-EWD monotherapy (Table 4). All of the monotherapy groups had initial diffuse oozing bleeding (Forrest lb), 
most of the lesions were $>1 \mathrm{~cm}(16 / 17,94.1 \%)$, with a different indication of cancer $(5 / 17,29.4 \%)$, post ESD or EMR bleeding $(10 / 17,58.8 \%)$, and ulcer $(2 / 17,11.8 \%)$. There was no rebleeding within 28 days of UI-EWD monotherapy. Moreover, no UI-EWD-associated adverse events, such as perforation or colonic obstruction, were recorded. 
Table 4

Bleeding characteristics and clinical outcome of patients in the U-EWD monotherapy group

\section{Variables}

Location of bleeding, $\mathrm{n}(\%)$

Hepatic flexure

Transverse colon

Sigmoid colon

Rectum

Cause of bleeding, $\mathrm{n}(\%)$

Ulcer bleeding

Post procedure bleeding

Tumor bleeding

Size of bleeding site, $\mathrm{n}(\%)$

$<1 \mathrm{~cm}$

$1-4 \mathrm{~cm}$

$>4 \mathrm{~cm}$

Forrest classification, $\mathrm{n}(\%)$

la

$\mathrm{lb}$

Cumulative re-bleeding, $\mathrm{n}(\%)$

At 7 days

At 14 days

At 28 days

Time to re-bleeding (days) $\S$

Adverse event, $\mathrm{n}(\%)$

$\S$ Median (range)

${ }^{+}$Fisher's exact test

${ }^{*} P$-values were calculated using the $t$-test or Fisher calculated using the of the conventional therapy group and the UI-EWD therapy group
UI-EWD monotherapy

$(n=17)$
$3(17.6)$

$4(23.5)$

$8(47.6)$

$2(11.8)$

$10(58.8)$

$5(29.4)$

$1(5.9)$

10 (58.8)

6 (35.3)

$0(0.0)$

17 (100.0)

$0(0.0)$

0 (0.0)

$0(0.0)$ 


\begin{tabular}{|ll|}
\hline Variables & $\begin{array}{l}\text { Ul-EWD monotherapy } \\
(\mathbf{n}=17)\end{array}$ \\
\hline Colonic obstruction & $0(0.0)$ \\
\hline Perforation & $0(0.0)$ \\
\hline Infection & $0(0.0)$ \\
\hline Embolization & $0(0.0)$ \\
\hline SMedian (range) & \\
\hline${ }^{+}$Fisher's exact test & \\
\hline $\begin{array}{l}{ }^{*} P \text {-values were calculated using the } t \text {-test or Fisher calculated using the of the conventional therapy } \\
\text { group and the Ul-EWD therapy group }\end{array}$ \\
\hline
\end{tabular}

\section{Discussion}

UI-EWD powder is a newly developed topical hemostatic agent. Use of this powder in refractory upper GI bleeding, non-variceal bleeding, and diffuse tumor bleeding has been described as safe and efficient for the prevention of rebleeding [18-21]. In the present study, we showed another promising result of UI-EWD on LGIB in that it prevented cumulative re-bleeding rate down to $5.5 \%$, showing a greater advantage in sites that are difficult to access by endoscopy or those that are larger with diffuse oozing bleeding.

The rebleeding rate in the UI-EWD group was $5.5 \%$, which is a considerable improvement compared to the rate of $12.8 \%$ observed with previously reported hemostatic powders in LGIB [16]. In addition to the common advantages of hemostatic powders, including easier accessibility and greater applicability to larger and diffuse oozing bleeding lesions [24, 25], differences in the composition of hemostatic powders might affect the hemostatic efficiency. Although there has been no head-to-head comparison, UI-EWD contains aldehyde dextran as a main component mixed with $\varepsilon$-poly amino acid at a 4:1 ratio, while other commercially available powders are mainly composed of polysaccharides [20]. These differences in UIEWD components are thought to lower the rebleeding rate by increasing the adhesion power from the Schiff base reaction through the cross-linking of amine and aldehyde dextran groups when they come into contact with moisture. In previous reports, the UI-EWD hydrogel was still present at $70.2 \%$ of the sprayed bleeding sites by second-look endoscopy at $24 \mathrm{~h}[20]$.

The characteristic of hemostatic powder working as locally, a shorter hemostatic effect is inevitable under the conditions of GI tract movement and food or feces passing. In addition, in clinical settings, it has been reported that the rebleeding rate further increases with time despite a high rate of successful initial hemostasis as a result of using hemostatic powder in upper and lower GI bleeding [26]. Moreover, 
there was a high rate of rebleeding after application of Hemospray (33.7\%), with similar rates for both esophagogastroscopies and colonoscopies (35.6\% vs. $23.1 \%$ ). Most reported rebleeding occurred within 7 days (86.2\%) [27]. However, in our report, the UI-EWD group did not show delayed rebleeding between 7 and 28 days after the initial successful hemostasis in LGIB. In our study, rebleeding was reported in three cases within 7 days; however, no rebleeding was reported after 7 days.

The medical records of the three patients with rebleeding were reviewed. The first patient showed rebleeding 1 day after endoscopic submucosal dissection of a 3-cm Yamada type III polyp diagnosed with rectal cancer. Owing to the taller (as opposed to wide) shape, there is a high possibility that a thick feeding vessel was hidden by the remnant submucosal injection, which can cause active arterial bleeding. The second patient showed rebleeding 4 days after initial hemostasis of a rectal ulcer with underlying liver cirrhosis and a low platelet count of 20,000. These high bleeding tendencies could not escape from the rebleeding. The last patient showed rebleeding 5 days after initial hemostasis of a transverse colon ulcer and took dual antiplatelet agents due to a history of cardiac stent insertion. In addition to the hemostasis failure of initial clipping on diffuse oozing bleeding ulcers, clipping itself might promote bleeding by GI movement. Although there were only three rebleeding cases, patients with a higher bleeding tendency or those who bled from the rectum. Further, a well-designed prospective study of various conditions that increase rebleeding is needed.

In the UI-EWD monotherapy group (Table 4), most of the applied cases were post-procedure or tumor bleeding $(15 / 17,88.2 \%)$, with sizes of bleeding site $>1 \mathrm{~cm}(16 / 17,94.1 \%)$. In these cases, additional endoscopic hemostasis was hesitated because of the higher risk of delayed perforation. Despite these difficult circumstances, a single application of UI-EWD seemed to be sufficient to prevent rebleeding and allow initial hemostasis. Moreover, there was no reported perforation or colonic obstruction due to the single use of UI-EWD. This is meaningful in that applying mono-treatment of hemostatic powder is less traumatic and may be a reliable option for active oozing bleeding with larger size, especially for post procedure or tumor bleeding in LGIB. This is compatible with the findings of our previous report on the effectiveness of UI-EWD for upper GI tumor bleeding [18].

In the comparison of bleeding characteristics between the conventional therapy group and the UI-EWD therapy group, UI-EWD was more commonly applied to the hepatic flexure. This is known as one of the most difficult areas to access or to fix the endoscopic position due to its sharp angulation, and more frequently has larger sizes $>1 \mathrm{~cm}$, even $\geq 4 \mathrm{~cm}$. Even under these adverse conditions, the UI-EWD group showed a significantly lower cumulative rebleeding rate within 28 days (5.5\%) than the conventional therapy group (17\%).

Considering the adverse events of visceral perforation and splenic infarction reported previously when using Hemospray [28], we also evaluated UI-EWD-related adverse effects, including embolism, intestinal obstruction, and allergic reaction, due to the characteristics of the powder. However, there were no reported cases in the UI-EWD group compared to perforation or infection reported by coagulation treatment in the conventional therapy group. In case of perforation and embolism by Hemospray, it was 
considered due to the high pressure when injecting powder, which induces a patient's risk of abdominal pain after applying Hemosrapy [16]. As a result of direct comparison of pressure between UI-EWD and Hemospray, UI-EWD was significantly lower at $7 \mathrm{psi}$ than at $37 \mathrm{psi}$. These low injection pressures may have contributed to the safety of UI-EWD.

Our study has some limitations. First, it was a retrospective analysis at a single-center hospital, which may not be generalizable. Second, the patients in whom UI-EWD was applied were not randomly distributed and the decision to use is left to the discretion of the physicians, which could present a selection bias. Despite these limitations, bleeding sites in the UI-EWD therapy group were more difficult to endoscopically approach, such as hepatic flexure or sigmoid colon, and were more common in the longer diameter group. To obtain further information on the practical performance of UI-EWD in LGIB and to evaluate the feasibility and efficacy of LGIB, a well-designed, large-scale, prospective study is needed.

In conclusion, this is the first study to demonstrate the effectiveness and safety of UI-EWD in the treatment of LGIB. Application of UI-EWD resulted in a high rate of immediate hemostasis (100\%) and a significant reduction in re-bleeding in patients with LGIB whose condition is not well controlled by conventional treatment ( $17 \%$ vs. $5 \%, p=0.044)$. Based on our results, we suggest that the use of UI-EWD for hemostasis in LGIB is sufficiently effective and safe.

\section{Declarations}

\section{Availability of data and materials}

The datasets used and analyzed during the current study are not publicly available due to keeping privacy of patients but are available from the corresponding author on reasonable request.

\section{Acknowledgments}

This work was supported by an INHA UNIVERSITY Research Grant.

\section{Funding}

Not applicable.

\section{Author information}

\section{Contributions}


JS conceptualized the project and made critical revisions to the paper. BC and DHL collected and assembly of data and wrote the initial manuscript draft. BC, DHL, JS, JP, KSK, and HK analyzed and interpretated data.

\section{Corresponding author}

Correspondence to Jongbeom Shin

\section{Ethics declarations}

\section{Ethics approval and consents to participate}

The study protocol was approved by the institutional review board of our institution (INHAUH 2020-03010). This was a retrospective study and patient consent was not required for data collection or analysis, as approved by the aforementioned institutional review board. All methods were carried out in accordance with relevant guidelines and regulations.

\section{Consent for publication}

Not applicable.

\section{Competing interests}

All authors have no competing interests to declare in connection with this study

\section{References}

1. Nabi Z. Complications of therapeutic gastroscopy/colonoscopy other than resection. Best Pract Res Clin Gastroenterol. 2016; 30(5):719-33.

2. Strate LL, Gralnek IM. ACG Clinical Guideline: Management of Patients With Acute Lower Gastrointestinal Bleeding. Am J Gastroenterol. 2016; 111(4):459-74.

3. Feinman M, Haut ER. Lower gastrointestinal bleeding. Surg Clin North Am. 2014; 94(1):55-63.

4. Qayed E, Dagar G, Nanchal RS. Lower Gastrointestinal Hemorrhage. Crit Care Clin. 2016; 32(2):24154.

5. Kwak MS, Cha JM, Han YJ, Yoon JY, Jeon JW, Shin HP, Joo KR, Lee JI. The Clinical Outcomes of Lower Gastrointestinal Bleeding Are Not Better than Those of Upper Gastrointestinal Bleeding. J Korean Med Sci. 2016; 31(10):1611-6. 
6. Ofosu A, Ramai D, Latson W, Adler DG. Endoscopic management of bleeding gastrointestinal tumors. Ann Gastroenterol. 2019; 32(4):346-51.

7. Hirasawa K, Sato C, Makazu M, Kaneko H, Kobayashi R, Kokawa A, Maeda S. Coagulation syndrome: Delayed perforation after colorectal endoscopic treatments. World J Gastrointest Endosc. 2015; 7(12):1055-61.

8. Holster IL, Kuipers EJ, Tjwa ET. Hemospray in the treatment of upper gastrointestinal hemorrhage in patients on antithrombotic therapy. Endoscopy. 2013; 45(1):63-6.

9. Babiuc RD, Purcarea M, Sadagurschi R, Negreanu L. Use of Hemospray in the treatment of patients with acute UGIB - short review. J Med Life. 2013; 6(2):117-9.

10. Prei JC, Barmeyer C, Bürgel N, Daum S, Epple HJ, Günther U, Maul J, Siegmund B, Schumann M, Tröger $\mathrm{H}$, et al. EndoClot Polysaccharide Hemostatic System in Nonvariceal Gastrointestinal Bleeding: Results of a Prospective Multicenter Observational Pilot Study. J Clin Gastroenterol. 2016; 50(10):e95-e100.

11. Beg S, Al-Bakir I, Bhuva M, Patel J, Fullard M, Leahy A. Early clinical experience of the safety and efficacy of EndoClot in the management of non-variceal upper gastrointestinal bleeding. Endosc Int Open. 2015; 3(6):E605-9.

12. Kurt M, Onal I, Akdogan M, Kekilli M, Arhan M, Sayilir A, Oztas E, Haznedaroglu I. Ankaferd Blood Stopper for controlling gastrointestinal bleeding due to distinct benign lesions refractory to conventional antihemorrhagic measures. Can J Gastroenterol. 2010; 24(6):380-4.

13. Garber A, Jang S. Novel Therapeutic Strategies in the Management of Non-Variceal Upper Gastrointestinal Bleeding. Clin Endosc. 2016; 49(5):421-4.

14. Facciorusso A, Straus Takahashi M, Eyileten Postula C, Buccino VR, Muscatiello N. Efficacy of hemostatic powders in upper gastrointestinal bleeding: A systematic review and meta-analysis. Dig Liver Dis. 2019; 51(12):1633-40.

15. Hookey L, Barkun A, Sultanian R, Bailey R. Successful hemostasis of active lower GI bleeding using a hemostatic powder as monotherapy, combination therapy, or rescue therapy. Gastrointest Endosc. 2019; 89(4):865-71.

16. Mourad FH, Leong RW. Role of hemostatic powders in the management of lower gastrointestinal bleeding: A review. J Gastroenterol Hepatol. 2018; 33(8):1445-53.

17. Holster IL, Brullet E, Kuipers EJ, Campo R, Fernández-Atutxa A, Tjwa ET. Hemospray treatment is effective for lower gastrointestinal bleeding. Endoscopy. 2014; 46(1):75-8.

18. Shin J, Cha B, Park JS, Ko W, Kwon KS, Lee JW, Kim HK, Shin YW. Efficacy of a novel hemostatic adhesive powder in patients with upper gastrointestinal tumor bleeding. BMC Gastroenterol. 2021; 21(1):40.

19. Bang B, Lee E, Maeng J, Kim K, Hwang JH, Hyon SH, Hyon W, Lee DH. Efficacy of a novel endoscopically deliverable muco-adhesive hemostatic powder in an acute gastric bleeding porcine model. PLoS One. 2019; 14(6):e0216829. 
20. Lee $\mathrm{D}, \mathrm{Ko}$ W. Hemostatic materials in non-variceal upper gastrointestinal hemorrhage. International Journal of Gastrointestinal Intervention. 2020; 9:1-3.

21. Park JS, Kim HK, Shin YW, Kwon KS, Lee DH. Novel hemostatic adhesive powder for nonvariceal upper gastrointestinal bleeding. Endosc Int Open. 2019; 7(12):E1763-e7.

22. Park JS, Bang BW, Hong SJ, Lee E, Kwon KS, Kim HK, Shin YW, Lee DH. Efficacy of a novel hemostatic adhesive powder in patients with refractory upper gastrointestinal bleeding: a pilot study. Endoscopy. 2019; 51(5):458-62.

23. Laine L, Shah A. Randomized trial of urgent vs. elective colonoscopy in patients hospitalized with lower GI bleeding. Am J Gastroenterol. 2010; 105(12):2636-41; quiz 42.

24. Savides TJ, Jensen DM, Cohen J, Randall GM, Kovacs TO, Pelayo E, Cheng S, Jensen ME, Hsieh HY. Severe upper gastrointestinal tumor bleeding: endoscopic findings, treatment, and outcome. Endoscopy. 1996; 28(2):244-8.

25. Barkun AN, Moosavi S, Martel M. Topical hemostatic agents: a systematic review with particular emphasis on endoscopic application in GI bleeding. Gastrointest Endosc. 2013; 77(5):692-700.

26. Haddara S, Jacques J, Lecleire S, Branche J, Leblanc S, Le Baleur Y, Privat J, Heyries L, Bichard P, Granval $P$, et al. A novel hemostatic powder for upper gastrointestinal bleeding: a multicenter study (the "GRAPHE" registry). Endoscopy. 2016; 48(12):1084-95.

27. Chahal D, Lee JGH, Ali-Mohamad N, Donnellan F. High rate of re-bleeding after application of Hemospray for upper and lower gastrointestinal bleeds. Dig Liver Dis. 2020; 52(7):768-72.

28. Yau AH, Ou G, Galorport C, Amar J, Bressler B, Donnellan F, Ko HH, Lam E, Enns RA. Safety and efficacy of Hemospray ${ }^{\circledR}$ in upper gastrointestinal bleeding. Can J Gastroenterol Hepatol. 2014; 28(2):72-6.

\section{Figures}




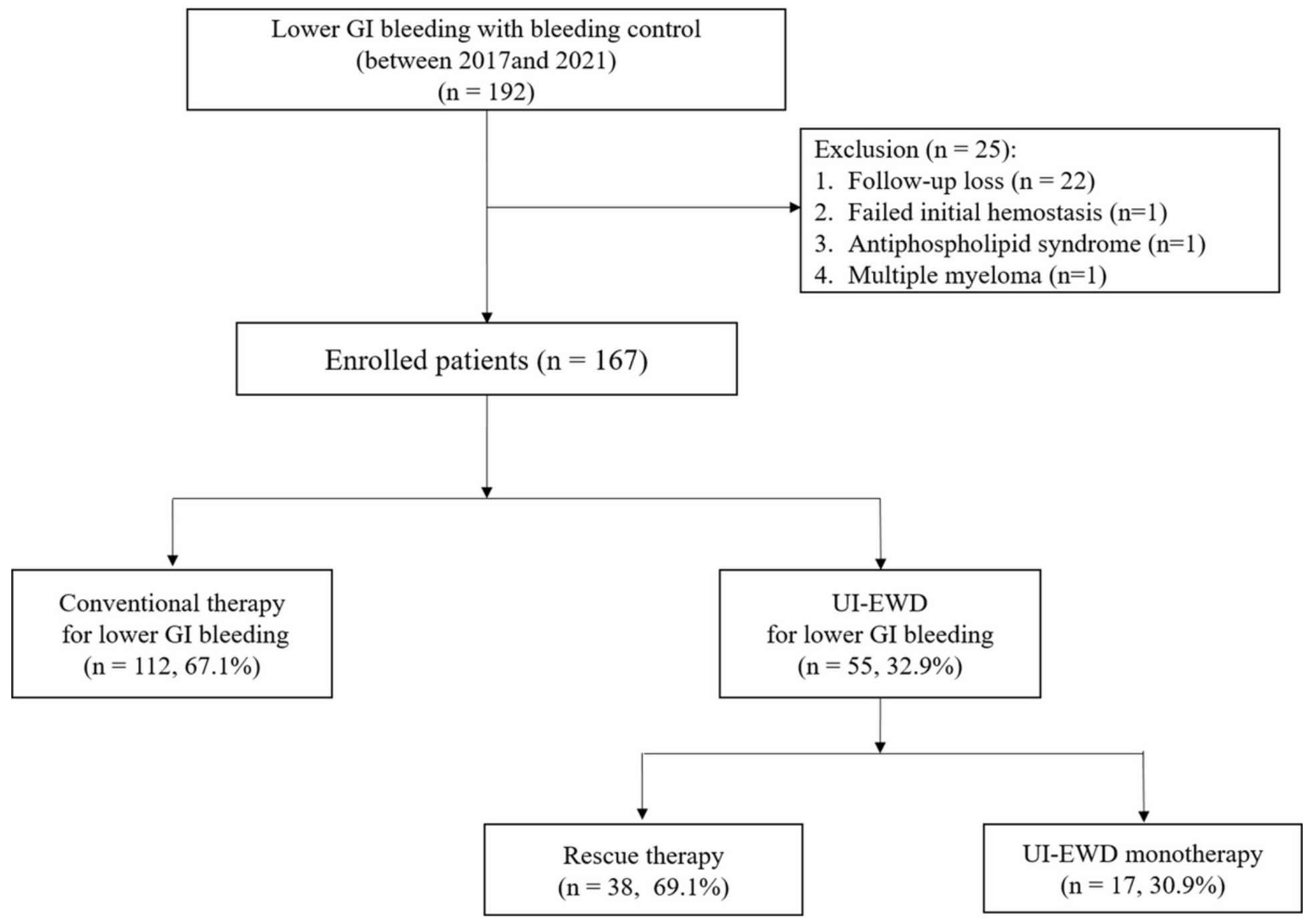

\section{Figure 1}

Patient selection flowchart of the study

A

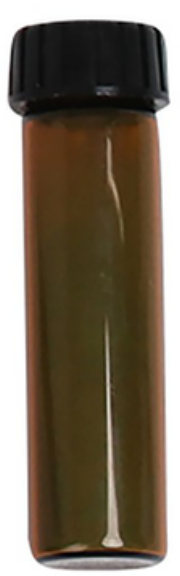

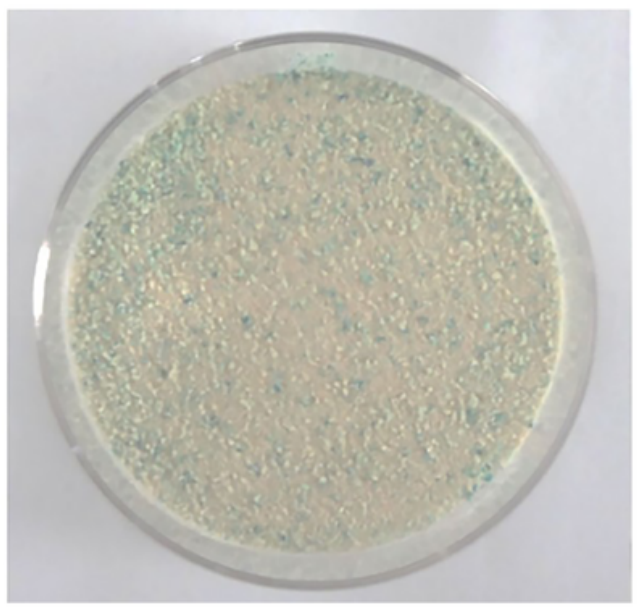

B

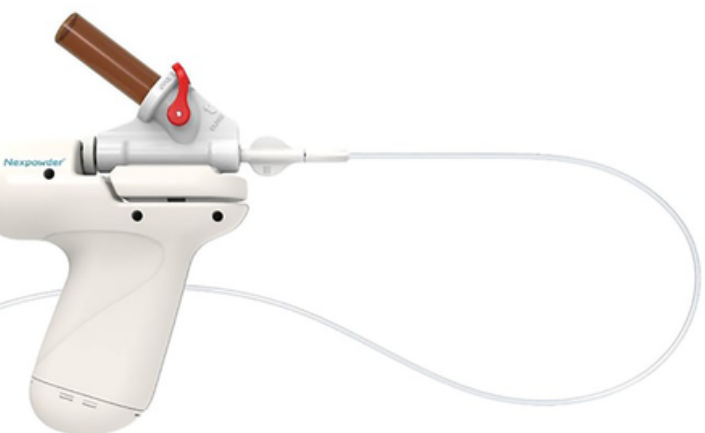

Figure 2 
UI-EWD (A) and powder delivery devices (B).
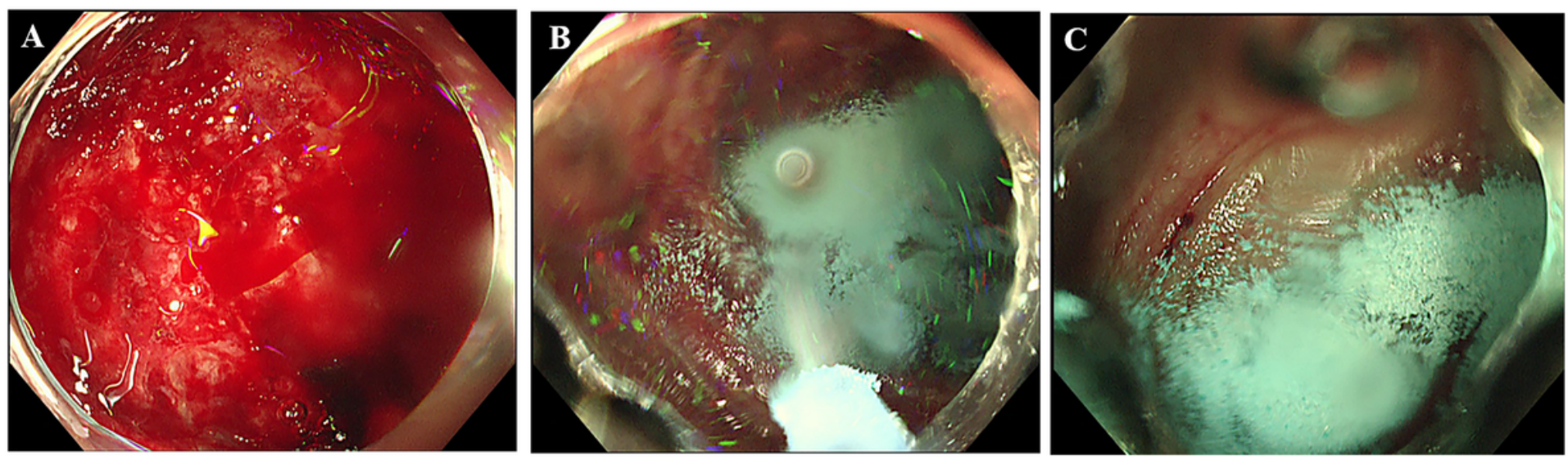

\section{Figure 3}

Endoscopic images of UI-EWD application in LGIB. (A) Case of diffuse oozing bleeding due to an ulcer on the hepatic flexure before the application of UI-EWD. (B) UI-EWD monotherapy was decided and applied due to anatomic difficulty in approaching by conventional treatment. (C) Successful hemostasis was confirmed $5 \mathrm{~min}$ after the application of the UI-EWD

A

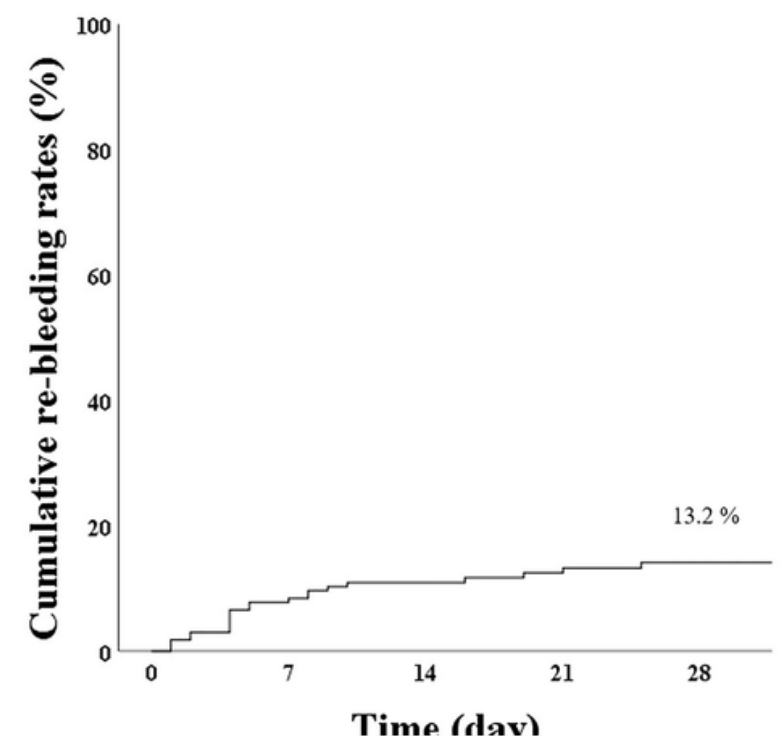

B

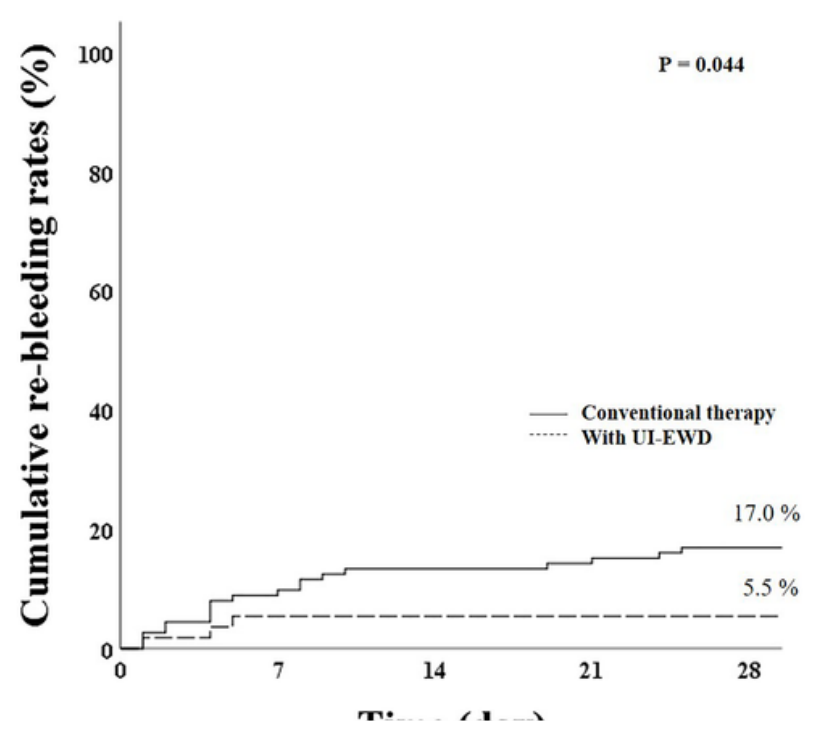

\section{Figure 4}

Cumulative re-bleeding rates in all enrolled patients $(A)$ and according to treatment modality $(B)$. 